\title{
The Imaging Informatics Response to a Pandemic
}

\author{
Ross W. Filice ${ }^{1}$ \\ Published online: 12 April 2021 \\ (c) Society for Imaging Informatics in Medicine 2021
}

Now that we are more than 1 year into the COVID-19 worldwide pandemic, we have seen many phases of diagnosis, response, and treatment broadly in medicine as well as in medical imaging. Radiology departments have been forced to respond and adapt to an unpredictable and variable landscape to continue providing necessary care to patients. This special edition highlights the use of imaging features to facilitate diagnosis of COVID-19 pneumonia and the prognosis for these patients; how technology solutions have helped hospitals adapt to a new social distancing, remote consultation, and pandemic landscape; and how to rapidly and successfully train radiologists to better detect COVID-19 pneumonia.

Early in the pandemic, when widespread PCR diagnostic testing was not available in hard-hit locations such as China and Iran, medical imaging was often the only means to triage patients under investigation for COVID-19. Fortunately, CT has been shown to be a sensitive but only slightly less specific triaging tool that can supplement and even substitute for PCR testing where needed [1]. Even as PCR and other forms of serological testing became widely available, CT and chest radiography imaging features were shown to continue to aid both diagnosis and prognosis [2, 3].

Radiology departments, as with many other specialties, found themselves having to adjust to the pandemic environment to both protect their staff and to ensure continuity of radiology services for patient care. Social distancing for both patient and staff proved to be challenging across many facets of hospital business continuity. As patient visits were staggered and moved to evenings and weekends, staffing models had to adapt to provide timely interpretation. Substantial amounts of image interpretation, and in the cases of academic institutions teaching and other trainee interaction, were moved to remote platforms which required rapid deployment of remote workstations to include technology that could facilitate this remote work [4-7]. This includes a remotely accessed web-based teaching application that was shown to be effective at improving radiologist performance in diagnosing this novel condition [8].

Finally, radiology departments were not the only imaging services that had to adapt to this new pandemic working environment. Dermatology departments, for example, shifted to support virtual evaluations as well [9].

The COVID-19 pandemic has been trying for all healthcare providers. Even with vaccines now in the early phases of distribution, the pandemic continues to rage worldwide. Imaging departments and imaging informatics technology have found successful paths of adaptation to ensure continuity of patient care.

\section{References}

1. Clinical application of chest computed tomography (CT) in detection and characterization of coronavirus (Covid-19) pneumonia in adults. https://doi.org/10.1007/s10278-021-00426-5

2. Novel chest radiographic biomarkers for COVID-19 using radiomic features associated with diagnostics and outcomes. https:// doi.org/10.1007/s10278-021-00421-w

3. Multicenter assessment of CT pneumonia analysis prototype for predicting disease severity and patient outcome. https://doi.org/ 10.1007/s10278-021-00430-9

4. Disaster planning during SARS-CoV-2/COVID: One Radiology Informatics Team's Story. https://doi.org/10.1007/ s10278-021-00420-x

5. The role of imaging informatics in disaster preparedness during the COVID-19 pandemic. In press

6. The radiology virtual reading room: During and beyond the COVID-19 pandemic. https://doi.org/10.1007/ s10278-021-00427-4

7. The new normal: Coronavirus pandemic response utilizing Microsoft SharePoint. https://doi.org/10.1007/s10278-021-00419-4

8. Developing a training web application for improving the COVID19 diagnostic accuracy on chest X-ray. https://doi.org/10.1007/ s10278-021-00424-7
Ross W. Filice

Ross.W.Filice@medstar.net

1 Georgetown University, Washington, DC, USA 
9. Supporting virtual dermatology consultation in the setting of COVID-19. https://doi.org/10.1007/s10278-021-00425-6
Publisher's Note Springer Nature remains neutral with regard to jurisdictional claims in published maps and institutional affiliations. 